\title{
CoNf- $721112--1$
}

RECEIVED BY TIC FEB 11973

LIQUID ENTRAINMENT RATE AND DROPLET SIZE RELATIVE TO FUEL FAILURE PROPAGATION DUE TO FISSION-GAS JET-IMPINGEMENT IN LMFBR SUBASSEMBLIES

T. C. Chawla

\author{
Presented at: \\ American Socjety of Mechanical Engineers \\ Winter Annual Meeting \\ New York, N. Y. \\ November 26-30, 1972
}

ASME Paper No. 72-WA/NE-7

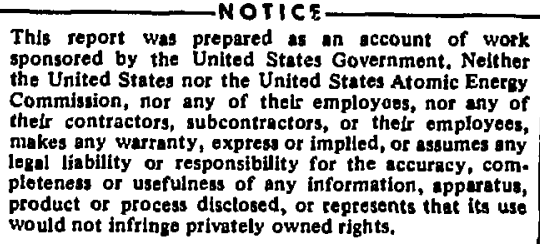

report was prepared as an account of work

United States Government. Nelther would not infringe privately owned rights. 


\section{Liquid Entrainment Rate and Droplet Size}

Relative to Fuel Failure Propagation Due to Fission-Gas Jet-Impingement in LMFBR Subassemblies

\section{T. C. CHAWLA}

Argonne National Laboratory, Reactor Analysis and Safety Div., Argonne, III., Assoc. Mem. ASME

The Kelvin-Helmholtz instability of the gas-liquid interface of sonic gas jet submerged in a mass of a viscous liquid is studied in order to determine the rate of liquid entrainment as well as the droplet size resulting from tearing off the liquid from the unstable liquid surface. The determination of the size of the droplets and the rate of liquid entrained is required in formulating a correlation for the heat transfer coefficient in the spray-cooled region of the fission-gas jet-Impingement area in LMFBR subassemblies. The linear hydrodynamic stability of the gasliquid interface under the action of the pressure perturbations, the interfacial surface tension, and the finite liquid viscous forces, is considered. For fission gas jets with values for the ratio between radius and wavelength of the disturbance much greater than one, a short-wave approximation to the dispersion equation for the instability is obtained. Approximate solutions for the growth parameters of the wave having maximum growth rate both for the case of inviscid liquid and highly viscous liquid are obtained; the exact solution for liquids having a finite viscosity is also presented. 


\title{
Liquid Entrainment Rate and Droplet Size
}

\section{Relative to Fuel Failure Propagation Due}

\section{to Fission-Gas Jet-Impingement in LMFBR}

\section{Subassemblies}

\author{
T. C. CHAWLA
}

\section{INTRODUCTION}

In the safety analysis of fast reactors that utilize nonvented fuels, the realistic assessment of the fallure potential of the fission gas released from a ruptured fuel pin near the end-oflife In the form of a persistent "choked" $(\underline{1})^{1}$ gas jet impinging on an opposite fuel pin cannot be made without an adequate means of estimation of

1 onderlined numbers in parentheses designate Feferences at end of paper:- sizes of droplets and the rate of coolant entrai ed as the jet passes through the coolant prier $t$ impinging on the opposite fuel pin. The liquid drops entrained at the gas-Iiquid interface produce a spray cooling of the opposite pin and thu govern the rate of heat transfer from that fuel pin. It may be noted that, without the spray cooling, the persistent gas jet, because it tends to reduce the rate of heat transf'er as cumpared Ifquid-metal coolant, could potentially lead to Nefure of the cladding of the opposite fueI pin

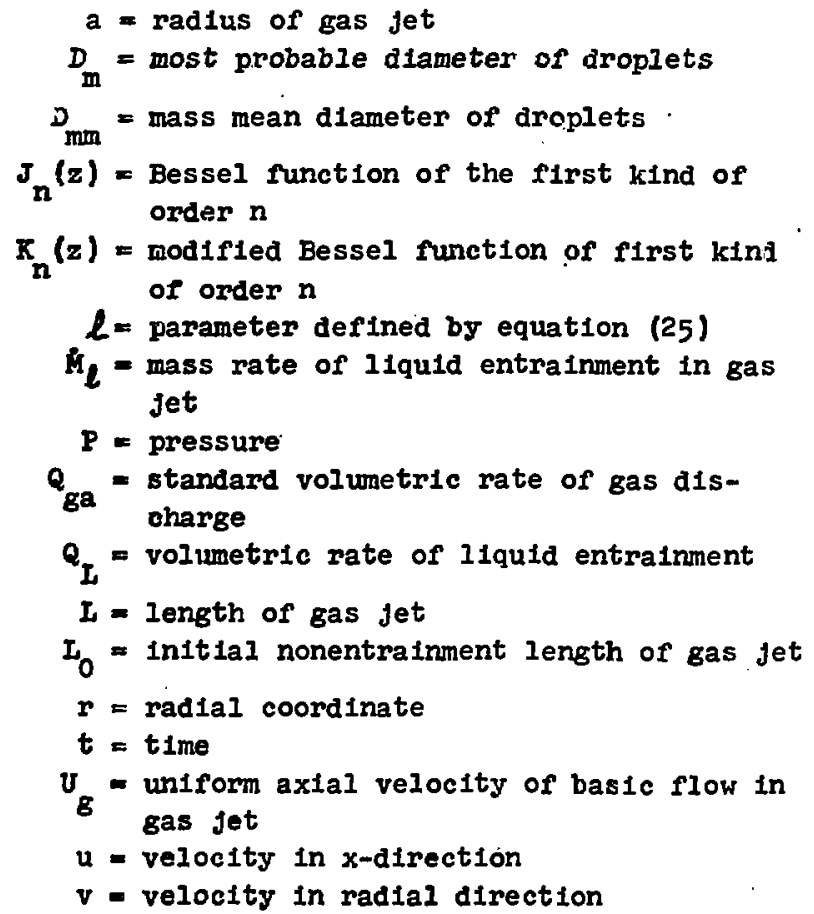

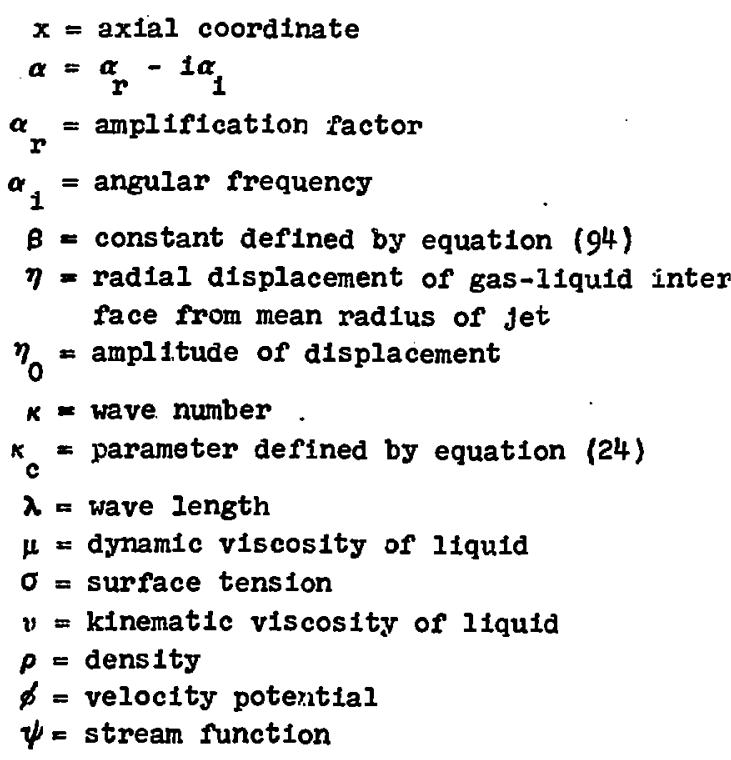


Thus, the prediction of the rate of liquid entrainment taking place at the surface of a gas jet submerged in a liquid is of considerable importance to IMFBR (Iiquid-Metal Fast Breeder Reactor) reactor safety.

The mechanism of liquid drop entrainment at the surface of the gas jet submerged in a liquid is governed by the Kelvin-Helmholtz instability of the gas-liquid interface. Several invesiigators have previously examined analytically the KelvinHelmholtz instabiiity of the gas-liquid interface. Chang and Russell (ㄹ) analyzed the case of a plane liquid layer exposed to subsonic and supersonic gas streams. Nactsheim (3) has considered the three-dimensional disturbance of a shear flow of a thin liquid film adjacent to a supersonic gas stream with wave fronts oblique to the external stream. A non-linear analysis of the KelvinHelmholtz instability of a liquid film adjacent tc a compressible gas and under the influence of a bod: force, directed either outward from or toward the Iiquid, has been presented by Nayfeh and Saric (4). Drazin (5) has analyzed the non-linear Kelvin-Helmholtz instability of two parallel horizontal streams of inviscid incompressible fluids. Numerous other studies, generally or an empirical nature, are concerned with the determination of entrainment rates in the gas core of gas-liquid cocurrent flows in a pipe. A bibliography is contained in Hewitt and Hall-Taylor (ㅁ).

In the present investigation, the linear stability of the gas-liquid interface of a sonic gas jet submerged in a mass of liquid is considered; the results of the analysis are applied to obtain the rate of liquid entrainment at the gasliquid interface and the size of droplets resulting from the breakup of liquid "rings" torn of $f$ the unstable gas-Iiquid interface by the sonic gas jet. one of the main difficulties in determining the effect of the sonic-flow (or transonic flow) regime in the round gas jet on the interfaclal stability of the liquid layer lies in arriving at an analytical description of the complicated flow structures of the gas jet. Experimental observations of Powell (I) indicate that the flow structure of the "critical" jet is periodic in the sense that the jet at first on leaving the orifice expands and then at a distance (which depends on the pressure ratiol from the orffice contracts to the diameter of orifice; conditions in the jet are then very similar to those existing at the orifice 1tself. Beyond this distance, the structure of the jet repeats Itself for several cycles. To describe the flow structure of the "critical" gas jet in all 1ts detalis is beyond the scope of the present study. Here, we merely would adopt, in part, a very simplified description shown by
Ovsiannikov (8) and Sedov (9) to be approximately valid. Theoretical findings of these authors indicate that for a jet issuing from a vessel with plane walls and the critical pressure occurring on the free stream, the jet achleves the sonic velocity at a finite distance from the vessel; beyond this distance, fJ.ow is continued approximately as a uniform flow with the sonic velocity. This description of the flow in the "critical" jet is necessarily valid (approximately) within only short lengths of jet, because the shear layer at the surface of the jet, though very thin in the beginning, gets thicker as the distance downstream from the orifice increases. In the present analysis for application to IMFBR reactor safety as discussed previously and liquid atomization (e.g., in pneumatic atomizers) in gas streams having sonic velocity, only short lengths of the gas jet are of interest (e.g., in LMFBR reactor safety, lengths of order 1.5 to $7 \mathrm{~mm}$ are involved). The expansion of the jet fluid as it leaves the orifice will be neglected. The jet is assumed to have a uniform sonic velocity and dameter equal to the orifice diameter over the length of interest; flow in the jet is assumed to be described by Iinearized compressible flow theory with neglecting the shear layer that exists near the boundary of the jet at the gas-liquid interface. The basis for neglecting the shear layer is that the pressure force, which is the dominant force as compared to the frictional force at the liquid surface, is not affected by the presence of the shear layer (2).

The choice of the foregoing model for the sonic gas jet was greatly influenced by Tam's work (10) on the study of directional acoustic radiation from a round, underexpanded supersonic gas jet. Tam neglected the initial expansion of the jet fluid and assumed the motion of the fluid in the core of the jet can be described by linearized potential flow theory with an infinitesimally thin shear layer on the surface of the jet. of course, in the present study, for reasons discussed previously, the thin shear layer on the surface of the sonic jet is neglected.

In the application of the instability analysis of the gas-liquid interface to the determination of the rate of liquid entrainment and droplet size, Mayer's postulated mechanism (11) for liquid breakup from an unstable liquid surface and Taylor's hypothesis (12) for the size of breakup are cf particular importance. Mayer postulated that the liquid is torn off from an unstable liquid surface at the wave crests by the high-speed gas stream in the form of "Iigaments" of Ilquid and these, still being unstable because of high ratio of surface energy to mass break up in drops. Tay- 


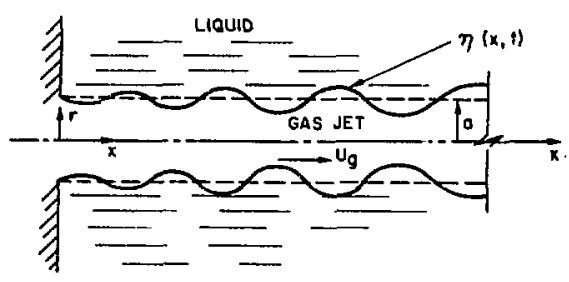

Fig.1 Description of the coordinate system

lor postulated that the most frequent size of drops formed from breakup of the liquid torn from an unstable liquid surface by an air stream is proportional to the wave length at the maximum amplification of the wave.

\section{FORMULATION}

The physical problem considered is shown in Fig. 1. The fisision-gas jet issues from a pinhole failure of radius $a$ in the cladding of a nonvented fuel pin with a gas-plenum pressure that the gas jet is "choked." (E.g., near the and-oflife of the fuel pin, a gas plenum pressure as high as 800 to 1000 psia may build up.) Furthermore, in the gas jet, only those fission-gas release rates are considered that lead to a persistent gas jet of sufficient duration so that the basic flok can be considered quasi-stationary; these rates depend on size of the breach, its axial location, and the impedance to the flow of gas internally in the pin during its passage to the breach. The fluid surrounding the jet is a liquid coolant. The effect of the initial coolant velocity transverse to the gas jet is neglected. This assumption is justified by the fact that the initial transverse (to the jet) coolant velocity becomes negligibly small due to the presence of the gas in the affected subchannels; the experimental results (13) also support this assumption.

In view of the forcoling assumptions and the previous discussion, the equations of motion for the gas fet with the basic flow having a uniform velocity, $U_{G}$, parallel to $x$-axis are

$$
\begin{aligned}
& \frac{\partial^{2} \phi_{g}}{\partial r^{2}}+\frac{1}{r} \frac{\partial \phi_{g}}{\partial r}-\frac{2}{U_{g}^{2}} \frac{\partial^{2} \phi_{g}}{\partial x \partial t}+\frac{1}{U_{g}^{2}} \frac{\partial^{2} \phi_{g}}{\partial t^{2}}=0 ; \\
& p_{g}=C_{g}(t)-p_{g}\left[\frac{\partial \phi_{g}}{\partial t}+\frac{1}{2} u_{g}^{2}+v_{g} \frac{\partial \phi_{g}}{\partial x}\right],
\end{aligned}
$$

where $\phi_{g}$ is velocity potential of the superposed transient motion, $1, e .$,

$$
u_{g}=u_{g}+\frac{\partial \phi_{g}}{\partial x} ; v_{g}=\frac{\partial \phi_{g}}{\partial r} ;
$$

$u_{g}$ and $v_{g}$ are the axial and radial components of the transient motion, respectively; $\mathrm{P}_{\mathrm{g}}$ is the pressure at a point in the gas jet; $C_{g}$ is a constant; $\rho_{g}$ is the gas density of the basic flow; $t$ is the time, and $r$ and $x$ are the coordinates of $a$ point. The transient motion of the surrounding coolant with a density, $\rho$, and kinematic viscosity, 1 , is represented by the following equations:

$$
\begin{gathered}
\frac{\partial u}{\partial x}+\frac{1}{r} \frac{\partial}{\partial r}(r v)=0 ; \\
\frac{\partial u}{\partial t}=-\frac{1}{\partial} \frac{\partial P}{\partial x}+v\left[\frac{\partial^{2} u}{\partial x^{2}}+\frac{1}{r} \frac{\partial}{\partial r}\left(r \frac{\partial u}{\partial r}\right)\right] ; \\
\frac{\partial v}{\partial t}=-\frac{1}{p} \frac{\partial P}{\partial r}+v\left\{\frac{\partial^{2} v}{\partial x^{2}}+\frac{\partial}{\partial r}\left[\frac{1}{r} \frac{\partial}{\partial r}(r v)\right]\right\},
\end{gathered}
$$

where $u$ and $v$ are axial and radial components of the induced motion of the liquid, respectively, and $P$ is the pressure at any point in the liquid.

The gas-liquid interface is displaced and set into motion according to the expression

$$
n(x, t)=\eta_{0} e^{i k x+a t}
$$

where $k$ is the wave number, $c$ is a complex number, the real part of which is the amplification factor, and the imaginary part is the angular frequency of the aisturbance.

At the gas-liquid interface, the radial components of gas and liquid velocities are equal to radial velocity of the interface; the latter is given by

$$
\frac{d n}{d t}=\frac{\partial n}{\partial t}+\frac{\partial n}{\partial x} \frac{d x}{d t} \quad \text { at } r=a \text {. }
$$

The halance of normal forces on the interface gives

$$
-P+2 \mu \frac{\partial v}{\partial r}+P_{g}=\sigma\left(\frac{1}{a}-\frac{n}{a^{2}}-\frac{\partial^{2} n}{\partial x^{2}}\right) \text { at } r=a \text {, }
$$

where $\mu$ is the dynamic viscosity of liquid and $\sigma$ is the surface tension. The balance of tangential forces at the interface gives

$$
u\left(\frac{\partial u}{\partial r}+\frac{\partial v}{\partial x}\right)-0 \text { at }:=a \text {. }
$$

In writing down the foregoing boundary condition, the effect of viscosity in the perturbed motion of the gas has been neglected. 
RESOLUTTON OF EQUATIONS OF MOTTON FOR THIE

\section{IIQUID PHASE}

Equations (4) and (5) can be simplified by use of a stream function, $\psi(r, x, t)$, defined as

$$
u=\frac{1}{r} \frac{\partial y}{\partial r} ; v=-\frac{1}{r} \frac{\partial \psi}{\partial x},
$$

which follows directly from the continuity equation (3). The substitution of the foregoing expressions into squations (4) and (5) yiejds

$$
\begin{aligned}
& \frac{1}{r} \frac{\partial}{\partial r}\left(\frac{\partial \psi}{\partial t}\right)=-\frac{1}{\rho} \frac{\partial P}{\partial x}+\frac{\nu}{r} \frac{\partial}{\partial r}\left[\frac{\partial^{2} \psi}{\partial x^{2}}+r \frac{\partial}{\partial r}\left(\frac{1}{r} \frac{\partial \psi}{\partial r}\right)\right] ; \\
& -\frac{1}{r} \frac{\partial}{\partial x}\left(\frac{\partial \psi}{\partial t}\right)=-\frac{1}{p} \frac{\partial P}{\partial r}-\frac{v}{r} \frac{\partial}{\partial x}\left[\frac{\partial^{2} \psi}{\partial x^{2}}+r \frac{\partial}{\partial r}\left(\frac{1}{r} \frac{\partial \psi}{\partial r}\right)\right] .
\end{aligned}
$$

Eliminating pressure $P$ between equations (10) and (11)

$$
\left(D-\frac{1}{v} \frac{\partial}{\partial t}\right) D_{\psi}=0
$$

where

$$
D \equiv \frac{\partial^{2}}{\partial x^{2}}+r \frac{\partial}{\partial r}\left(\frac{1}{r} \frac{\partial}{\partial r}\right)
$$

Since the operators, (D - $\partial / \mu \partial t)$ and $D$, commute, the function, $\psi$, can be separated into two parts, of which $\psi_{1}$ satisfies

$$
D \psi_{1}=0
$$

and $\psi_{2}$ satisfies

$$
D \psi_{2}-\frac{1}{v} \cdot \frac{\partial \psi_{2}}{\partial t}=0
$$

and the solutions of equations (13) and (14) can then be combined to give the general solution of equation (12) as

$$
v=v_{1}+v_{2}
$$

Substituting equation (15) into equations (10) and (11), and using equations (13) and (14), we obtain

$$
\frac{1}{\partial} \frac{\partial P}{\partial X}-\cdot \frac{1}{r} \frac{\partial}{\partial r}\left(\frac{\partial v_{1}}{\partial t}\right) ;
$$

$$
\frac{1}{\partial T} \frac{\partial P}{\partial T}=\frac{1}{T} \frac{\partial}{\partial x}\left(\frac{\partial \psi_{1}}{\partial T}\right) .
$$

\section{SOLUTIONS}

We seek solutions or equations (1), (13), and (14) in the following form, respcctively,

$$
\begin{aligned}
& \phi_{g}(r, x, t)=\phi_{g}(r) e^{i k x+a t} ; \\
& \psi_{1}(r, x, t)=\psi_{1}(r) e^{i k x+a t} ; \\
& \psi_{2}(r, x, t)=\psi_{2}(r) e^{i k x+a t} .
\end{aligned}
$$

The substitution of the foregoing solutions into the respective equations yields

$$
\begin{aligned}
& \frac{d^{2} \phi_{g}(r)}{d r^{2}}+\frac{1}{r} \frac{d \phi_{g}(r)}{d r}+k_{c}^{2} \phi_{g}(r)=0 ; \\
& \frac{d^{2} \psi_{1}(r)}{d r^{2}}-\frac{1}{r} \frac{d \psi_{1}(r)}{d r}-k^{2} \psi_{1}(r)=0 ; \\
& \frac{d^{2} \psi_{2}}{d r^{2}}-\frac{1}{r} \frac{d \psi_{2}(r)}{d r}-\ell_{2}^{2} \psi_{2}(r)=0
\end{aligned}
$$

whei'e

$$
\kappa_{c}^{2}=\cdot \kappa^{2}\left[\frac{2 i \alpha}{\mathrm{U}^{k}}+\frac{\alpha^{2}}{U_{g}^{2} k^{2}}\right]
$$

and

$$
\ell^{2}=k^{2}+\frac{\alpha}{v}
$$

The solution of equation (21) that remains finite at the jet axis is

$$
\phi_{g}(r)=A_{1} J_{0}\left(k_{c} r\right)
$$

The solution of each of equations (22) and (23) that separately vanishes at infinity are, respectively,

$$
\begin{aligned}
& \psi_{1}(r)=A_{2} r K_{1}(x r) \\
& \psi_{2}(r)=A_{3} r K_{1}(l r) .
\end{aligned}
$$

Finally, we obtain

$$
\begin{aligned}
& \phi_{g}(r, x, t)=A_{1} J_{0}(k, r) e^{i \kappa x+a t}, \\
& \psi_{1}(r, x, t)=A_{2} r K_{1}(k r) e^{i \kappa x+a t}, \\
& \psi_{2}(r, x, t)=A_{3} r K_{1}(l r) e^{i k x+a t},
\end{aligned}
$$

and 


$$
v=\psi_{1}+\psi_{2}=\left[A_{2} K_{1}(\kappa v)+A_{3} K_{1}(\ell r)\right] r e^{i k x+a t} .
$$

From definitions of the velocity potential and stream function given previously and from the use of foregoing equations, we obtain

$$
\begin{gathered}
u_{g}=U_{g}+i k A_{1} J_{0}\left(k_{c} r\right) e^{i k x+a t} ; \\
v_{g}=-\kappa_{c} A_{2} J_{1}\left(\kappa_{c} r\right) e^{i \kappa x+a t} ; \\
u=-\left[k A_{2} K_{0}(k r)+l A_{3} K_{0}(l r)\right] e^{i k x+a t} ; \\
v=-i \kappa\left[A_{2} K_{1}(k r)+A_{3} K_{1}(l r)\right] e^{i k x+a t} .
\end{gathered}
$$

The substitution of equation (6) into equation (7) and equating the resultant equation to equation (34) gives

$$
\eta_{0}\left(a+i_{k} U_{g}\right)=-\kappa_{c} A_{1} J_{1}\left(\kappa_{c} a\right) \text { at } r=a \text {; }
$$

similarly, from the use of equations $(6),(7)$, and (36), we obtain

$$
a n_{0}=-i_{k}\left[A_{2} K_{1}(k a)+A_{3} K_{1}(l a)\right] \text { at } r=a .
$$

Substituting equations (35) and (36) into equation (9) yields

$$
2 A_{2} k^{2} K_{1}(k a)+A_{3}\left(k^{2}+\ell^{2}\right) K_{1}(l a)=0 \text { at } r=a
$$

The solutions of equations (37) to (39) gives

$$
\begin{gathered}
A_{1}=-\frac{i k U_{g}+a}{\kappa_{c} J_{2}\left(\kappa_{c} c^{2}\right)} n_{0}, \\
A_{2}=-\frac{i a}{k} \frac{k^{2}+\ell^{2}}{k^{2}-l^{2}} \frac{n_{0}}{K_{l}(k a)} .
\end{gathered}
$$

and

$$
A_{3}=\frac{2 i_{k a}}{\kappa^{2}-l^{2}} \frac{n_{0}}{K_{1}(a)} .
$$

The substitution of equation (29) with coefficient $A_{1}$ given by equation $(40)$ into equation (2) yields

$$
p_{g}=c_{g} \cdot \frac{1}{2} \rho_{g} U_{g}^{2}+\rho_{g}\left[a+i \kappa U_{g}\right]^{2} \frac{J_{0}\left(\kappa_{c} r\right)}{\kappa_{c} J_{1}\left(\kappa_{c} a\right)} \eta_{0} e^{i k x+a t} .
$$

The use of equation (30) with coefficlent $A_{2}$ given by equation (41) into equation (16) or (17) and integrating subsequently gives

$$
P=C-\rho a^{2} \frac{k^{2}+\ell^{2}}{\kappa^{2}-\ell^{2}} \frac{K_{0}(k r)}{k K_{1}(k a)} \eta_{0} e^{i k x+a t} .
$$

The constants, $\mathrm{C}_{\mathrm{g}}$ and $\mathrm{C}$, that occur in equations (43) and (44), respectively, are evaluated by applying equation (8) at; $x=0$ (i.e., at the location where the disturbance originates, and, as such at $x=0$, the deviation $\eta$ of the gas-liquid interface is zero). Thus, the substitution of equation (36) [with coefficients $A_{2}$ and $b_{3}$ given by equations (41) and (42), respectively], equations (43), and (44) into equation (8), and then dropping the terms that involve $\eta$, gives

$$
C_{g}=\frac{a}{a}+c+\frac{1}{2} \rho_{g} U_{g}^{2}
$$

Finally, with constant $c_{g}$ given by equation (45), and by substitution of equations (6), (36), (43), and (44) with constants $A_{2}$ and $A_{3}$ given by equations (41) and (42), respectively, into equation (8), we obtain

$$
\begin{aligned}
& \rho a^{2} \frac{k^{2}+\ell^{2}}{\kappa^{2}-\ell^{2}} \frac{K_{0}(k a)}{k K_{1}(k a)}+2 \mu \alpha\left\{\left(\frac{k^{2}+\ell^{2}}{\kappa^{2}-\ell^{2}}\right)\left[\frac{1}{a}+\frac{\kappa K_{0}(k a)}{K_{1}(k a)}\right]-\frac{2 k^{2}}{\kappa^{2}-\ell^{2}}\right. \\
& \left.\times\left[\frac{1}{a}+\frac{\ell K_{0}(l a)}{K_{1}(l a)}\right]\right\}+\rho_{g}\left(i \times U_{g}+a\right)^{2} \frac{J_{g}\left(\kappa_{c} a\right)}{K_{c_{1}} \kappa_{1}\left(\kappa_{c} c^{a}\right)}=\left(\frac{k^{2} a^{2}-1}{a^{2}}\right) 0 .
\end{aligned}
$$

\section{THE ASYMP'TOTIC APPROXIMATION}

The dispersion equation ( 46 ) is highly complex and cannot be solved analytically for $\alpha$. However, by employing asymptotic expansions of the Bessel functions for large and small arguments, equation (46) can be simplified considerably. The case of large arguments, i.e., $\mathrm{Ka} \gg \mathrm{I},|\boldsymbol{L}| \mathrm{a} \gg \mathrm{I}$, and $\left|\kappa_{c}\right| a \gg 1$, Implies that we consider only the contribution of short waves; the case of small argument implies contribution of only long waves. The only approximation relevant to liquid breakup by a high-speed gas jet at the gas-liquid interface is that of short waves. The long-wave length approximation is relevant to other jet phenomena involving scale lengths of the order of the jet diameter (10), e.g., breakup of a liquid jet at low flow velocity (24). Thus, for $\kappa a \gg 1$, $|\ell| x a \gg 1$, and $\left|\kappa_{c}\right| a \gg 1$, we employ the following asymptotic expansions (15): 


$$
\begin{gathered}
J_{n}(z) \sim \frac{1}{\sqrt{(2 \pi z)}}\left\{\exp \left[i\left(z-\frac{2 n+1}{4} \pi\right)\right]\right. \\
\left.+\exp \left[-i\left(2-\frac{2 n+1}{4} \pi\right)\right]\right] ; \\
K_{n}(z) \sim \sqrt{\frac{\pi}{2 z}} e^{-z} .
\end{gathered}
$$

The neglected terms in these asymptotic expansions are of the order of $(1 /|z|)$.

The asymptotic expression for $J_{n}(z)$ can be simplified further. If for a gas-liquid system under consideration, we make a prior assumption that $(|\alpha| / \kappa)$ is much smaller than $U_{g}$, the secondorder term in expression (24) for $\kappa_{c}$ can be neglected, with $\alpha$ expressed as

$$
a=a_{r}-i a_{i}
$$

(where $\alpha_{\text {, }}$ is the time amplification factor and $\alpha_{i}$ is the angular erequency of the disturbancel, and, by taking the principal root of equation (24), we obtain

$$
\kappa_{c}=\sqrt{\frac{k}{\sigma_{g}}}\left\{\left[\sqrt{a_{i}^{2}+a_{r}^{2}}-a_{i}\right]^{1 / 2}-i\left[\sqrt{a_{i}^{2}+a_{r}^{2}}+a_{i}\right]^{\frac{1}{2}}\right\} .
$$

Ciearly,

$\ln \left(x_{c}\right)=\sqrt{\frac{\alpha}{\sigma_{g}}}\left[\sqrt{a_{i}^{2}+a_{r}^{2}}+a_{i}\right]^{\frac{1}{2}}>R\left(x_{c}\right)=\sqrt{\frac{k}{\sigma_{g}}}\left[\sqrt{a_{i}^{2}+a_{r}^{2}}-a_{i}\right]^{\frac{1}{2}}>0 ;$ with $a\left|k_{c}\right| \gg 1$

$$
\operatorname{aIm}\left(\kappa_{\mathrm{c}}\right) \gg 1 .
$$

Substituting equation (50) into equation (47) and making ise of equation (51), we obtain the following simpler asymptotic formula for $J_{n}\left(k_{c} a\right)$ :

$$
J_{n}\left(x_{c} a\right) \sim \frac{1}{\left(2 \pi x_{c}\right)^{\frac{1}{2}}} \exp \left[i\left(\kappa_{c}^{a}-\frac{2 n+1}{a} n\right)\right]
$$

The substitution of equations (48) and (52) Into equation (46), and employing $U_{k} \gg|\alpha| / k$ and the short-wave approximation, we obtain

$$
\frac{k^{2}+\ell^{2}}{k^{2}-l^{2}} a^{2}+2 v k^{2} a \frac{(k-l)^{2}}{k^{2}-l^{2}}-i \frac{\rho_{q} k^{3}}{\partial k_{c}} u_{g}^{2}=\frac{g k^{3}}{p} .
$$

For ease in obtaining roots, equation (53) is separated into real and Imaginary parts by substituting for $l, \alpha$, and $\kappa_{c}$ from equations (25), (49), and (50), respectively; the resultant equations are

$$
\begin{aligned}
& -4 v k^{2} a_{r}+a_{i}^{2}-a_{r}^{2}-4 v^{2} k^{4}+2^{3 / 2} v^{2} k^{3}\left[\sqrt{\left(k^{2}+\frac{a_{r}}{v}\right)^{2}+\left(\frac{a_{i}}{v}\right)^{2}}\right. \\
& \left.+\left(k^{2}+\frac{a_{T}}{v}\right)\right]^{\frac{1}{2}}+\frac{\rho_{g}}{2 p}\left(k_{g}\right)^{5_{\Sigma}}\left[\frac{\sqrt{\alpha_{1}^{2}+\alpha_{r}^{2}}+\alpha_{i}}{\alpha_{i}^{2}+\alpha_{r}^{2}}\right]^{\frac{1}{2}}=\frac{\sigma k^{3}}{\sigma} ; \\
& 4 v k^{2} a_{i}+2 a_{i} a_{r}-2^{\frac{3}{2}} v^{2} k^{3}\left[\sqrt{\left(k^{2}+\frac{a}{v}\right)^{2}+\left(\frac{a_{i}}{v}\right)^{2}} \cdot\left(k^{2}+\frac{a_{r}}{v}\right)\right]^{\frac{4}{2}} \\
& -\frac{p_{g}}{2 \rho}\left(k U_{g}\right)^{\frac{5}{2}}\left[\frac{\sqrt{a_{i}^{2}+a_{r}^{2}}-a_{i}}{a_{i}^{2}+a_{r}^{2}}\right]^{\frac{1}{2}}=0 \text {. }
\end{aligned}
$$

GROWTH PATE OF THE INSTABILITY

The growth of the ampli.tude of the waves with time is represented by

$$
E(t)=\pi_{0} e^{R(a) t}=n_{0} e^{a} r^{t}
$$

The most rapid growth of the amplitude corresponds to the maximum value of $t_{p}(x)$ (with respect to wave number) for a given set of flow parameters. The maximum of $\alpha_{p}(k)$ is obtained by simultaneous solution of equations (54) and (55), and the following equation, which is obtained by differentiating equations (5 ) and (55) with respect to wave number $k$, setting $d a_{r} / d k=0$, and eliminating $\mathrm{d} \alpha_{1} / \mathrm{d} \kappa$ from the resultant equations:

$$
\begin{aligned}
& \int 8 v x_{r}+16 v^{2} k^{2}-6 \sqrt{2} v^{2} k l_{r}-\frac{2 \sqrt{2} v^{2} k^{3} l r}{\sqrt{\left(k^{2}+a_{I} / v\right)^{2}+\left(a_{i} / v\right)^{2}}}+\frac{3 g k}{p}
\end{aligned}
$$

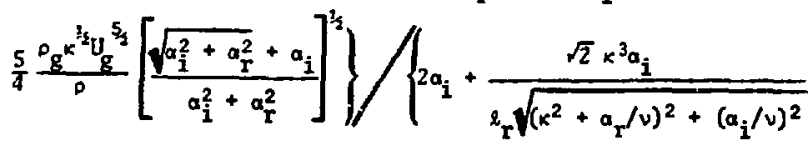

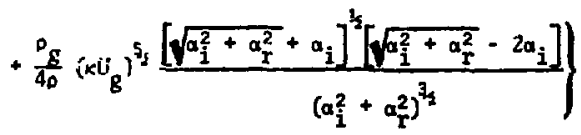

$$
\begin{aligned}
& -\left\{-8 v a_{i}+6 \sqrt{2} v^{2} k l_{i}+\frac{5}{4} \frac{\rho g k^{1 / 2 u_{g}^{5}}}{p}\left[\frac{\sqrt{\alpha_{i}^{2}+a_{r}^{2}}-a_{i}}{a_{i}^{2}+a_{r}^{2}}\right]^{\frac{1}{2}}\right. \\
& \left.\frac{2^{3} k^{3} v^{2} \ell_{i}}{\sqrt{\left(x^{2}+a_{r} / v\right)^{2}+\left(a_{i} / v\right)^{2}}}\right\} / 2 \alpha_{r}+4 v x^{2}-\frac{\sqrt{2} a_{i} k^{3}}{\ell_{i} \sqrt{\left(k^{2}+a_{r} / v\right)^{2}+\left(a_{i} / v\right)^{2}}} \\
& \left.+\frac{\rho_{g}\left(x u_{g}\right)^{\frac{5}{2}}}{\alpha_{p}} \frac{\left[\sqrt{a_{i}^{2}+a_{r}^{2}}-a_{i}\right]^{\frac{1}{2}}\left[\sqrt{a_{i}^{2}+a_{r}^{2}}+2 \alpha_{i}\right]}{\left(a_{i}^{2}+a_{r}^{2}\right)^{\frac{3}{3}}}\right\} \text {. }
\end{aligned}
$$


where

$$
\begin{aligned}
& e_{r}=\left[\sqrt{\left(k^{2}+a_{r} / v\right)^{2}+\left(\alpha_{i} / v\right)^{2}}+\left(k^{2}+\alpha_{r} / v\right)\right]^{1 / 5}, \\
& e_{i}=\left[\sqrt{\left(k^{2}+a_{r} / v\right)^{2}+\left(\alpha_{i} / v\right)^{2}}-\left(k^{2}+\alpha_{r} / v\right)\right]^{k} .
\end{aligned}
$$

For a given set of flow parameters, the simultaneous solution of equations (54) to (56) will yield three unknowns, viz., $\alpha_{r}, \alpha_{i}$, and $\kappa_{\text {. These sets }}$ of equations are quite complex, and an analyical solution, except for simple cases of low-viscosity and high-viscosity liquids (to be discussed subsequently), is not f'easible. However, prior to embarking on the numerica solution of equations (54) to (56), it is convenient to obtain solutions for simple cases of low-viscosity and high-viscosity liquids.

\section{Iow-Viscosity Liquid Approximation}

The low-viscusity Iiquid is defired as one for which the following inequality holds (14).

$$
|a| \gg v k^{2} \text { or }|e| \gg x \text {, }
$$

which leads to the foilowine simplified form of equations (54) and (55) for the unstable mode of disturbance:

$$
\begin{aligned}
& a_{i}^{2}-a_{r}^{2}+\frac{\rho g}{2 \rho}\left(k U_{g}\right)^{\frac{5}{2}}\left[\frac{\sqrt{\alpha_{i}^{2}+a_{r}^{2}}+a_{i}}{a_{i}^{2}+\alpha_{r}^{2}}\right]^{l_{1}}=\frac{\sigma k^{3}}{\rho}, \\
& 2 a_{i}=\frac{\rho_{g}}{2 \rho} \cdot \frac{\left(k U_{g}\right)^{\frac{5}{2}}}{\left[\left(a_{i}^{2}+\alpha_{r}^{2}\right)\left(\sqrt{\alpha_{i}^{2}+a_{r}^{2}}+a_{i}\right)\right]^{\frac{1}{2}}}=0,
\end{aligned}
$$

and the equation corresponding to equation (56) for low-viscosity liquid is ob'iained more simply from equations (58) and (59) $2 s$

$$
\frac{3 a_{i}\left(\alpha_{i}^{2}+\alpha_{r}^{2}\right)^{\frac{1}{2}}+2 a_{i}^{2}+a_{r}^{2}}{4 a_{i}^{2}+2 a_{r}^{2}+a_{i} \sqrt{a_{i}^{2}+\alpha_{r}^{2}}}=\frac{3 \sigma k^{3}}{\rho} \frac{\left[\sqrt{\alpha_{i}^{2}+a_{r}^{2}}+\alpha_{i}\right]^{\frac{1}{2}}}{\frac{5}{2} \frac{p_{g}}{\rho}\left(\alpha U_{g}\right)^{5 / 2}} .
$$

The use of equations (58) and (59) into equation (60) yielas

$$
\frac{3 a_{i}\left(a_{i}^{2}+a_{r}^{2}\right)^{4}+2 a_{i}^{2}+a_{r}^{2}}{4 a_{i}^{2}+2 a_{r}^{2}+a_{i}\left(a_{i}^{2}+a_{r}^{2}\right)^{\frac{1}{2}}}=\frac{3\left[3 \alpha_{i}^{2}-\alpha_{r}^{2}+2 \alpha_{i}\left(\alpha_{i}^{2}+a_{r}^{2}\right)^{\frac{1}{2}}\right]}{10 a_{i}\left(\alpha_{i}^{2}+\alpha_{r}^{2}\right)^{\frac{1}{2}}}
$$

which, after some algebraic manipulations, reduces to

$$
25\left(a_{i} / a_{r}\right)^{9}+575\left(a_{i} / a_{r}\right)^{6}-205\left(a_{i} / a_{r}\right)^{4}-215\left(a_{i} / a_{r}\right)^{2}-36.0=0 .
$$

This polynomiad equation is solved numerlcally, and the resultant solution that also satisfies equations (58) and (59) simultaneously is

$$
\alpha_{i}=0.92245661 \alpha_{r}
$$

Substituting equation (63) into equations (58) and (59), and solving for $\kappa, \alpha_{r}$, and $\alpha_{i}$, we obtain

$$
\begin{aligned}
& \kappa_{m}=0.80327205\left(\frac{\rho}{\rho_{g}}\right)^{0.2} \frac{\rho_{g} U_{g}^{2}}{\sigma} ; \\
& a_{\mathrm{rm}}=0.35717694\left(\frac{\rho_{g}}{\rho}\right)^{0.2} \frac{\rho_{g} U_{g}^{3}}{\sigma} ; \\
& a_{\mathrm{im}}=0.32948022\left(\frac{\rho_{g}}{\rho}\right)^{0.2} \frac{\rho_{g} U_{g}^{3}}{\sigma} .
\end{aligned}
$$

In the foregoing set of equations, the subscript, $m$, has been added to denote the values of $\kappa$ and $\alpha_{i}$ at the maximum value, $\alpha_{\mathrm{rm}}$, of the amplification factor of the waves. It may be noted that the expressions (64) to (66) can only be applied if inequality (57), or, which when expressed as follows in terms of flow parameiers by use of equations (64) to (66), i.e.,

$$
\left(\frac{p}{p_{g}}\right)^{0.4}\left(\frac{\sigma}{\mu U_{g}}\right) \gg 1,
$$

is satisfied.

High-Viscosity Liguid Approximation

A highly viscous Iiquid is defined by (14)

$$
|a| \ll v^{2} \text { or }|2|=k \text {. }
$$

The application of the foregoing approxtmation to equations (54) and (55) yields

$$
\begin{aligned}
& -2 v \alpha_{r}+\frac{\rho_{g}}{2 \rho} x^{\frac{1}{2}} u_{g}^{5_{s}} \frac{\left[\sqrt{\alpha_{i}^{2}+\alpha_{r}^{2}}+\alpha_{i}\right]^{1 / 2}}{\sqrt{\alpha_{i}^{2}+\alpha_{r}^{2}}}=\frac{\sigma k}{\rho} ; \\
& 2 v a_{i}-\frac{\rho g}{2 p} \kappa^{\frac{1}{2}} U_{8} \frac{\left[\sqrt{\alpha_{i}^{2}+a_{r}^{2}}-\alpha_{i}\right.}{\sqrt{\sigma_{1}^{2}+a_{r}^{2}}}=0 \text {, }
\end{aligned}
$$

and the maximum rate of growth of instability for high-viscosity liquid is obtalned from equations (69) and (70) as 


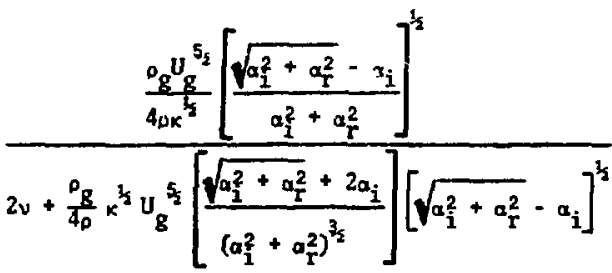

$$
\begin{aligned}
& =\frac{\frac{\sigma}{\rho} \sqrt{a_{1}^{2}+a_{I}^{2}}}{\frac{2 v}{a_{r}}\left[2 x_{1}^{2}+a_{T}^{2}+2 a_{i} \sqrt{a_{1}^{2}+a_{r}^{2}}\right]} .
\end{aligned}
$$

The substitution of equations (69) and (70) into equation (71) yields, after some algebraic manipulations,

$$
-27\left(\alpha_{i} / \alpha_{T}\right)^{6}-3\left(a_{i} / \alpha_{T}\right)^{4}+12\left(a_{i} / \alpha_{T}\right)^{2}+4=0 .
$$

The solution of this polynomial equation that also satisfies equations $(69)$ and (70) simultaneously is

$$
\alpha_{i}=0.86483545 \alpha_{r}
$$

Substitution of the foregoing into equations (69) and (70) yields

$$
\begin{aligned}
\kappa_{\mathrm{m}} & =0.35191129 \sqrt{\frac{\mu}{\sigma}} \frac{\rho_{\mathrm{g}} \mathrm{U}_{\sigma}^{5 / 2}}{\sigma} ; \\
\alpha_{\mathrm{m}} & =0.19740649 \frac{\rho_{\mathrm{g}} \mathrm{U}_{\mathrm{g}}^{\frac{5}{2}}}{\sqrt{\mu \sigma}} ; \\
\alpha_{\mathrm{im}} & =0.17072413 \frac{\rho_{\mathrm{g}} U_{\mathrm{g}}^{5 / 2}}{\sqrt{\mu \sigma}} .
\end{aligned}
$$

Substituting for $|\alpha|$ and $\alpha$ from the foregoing set of equations into equation (68), we obtain the corresponding condition for which above expressions are valid:

$$
\frac{\rho}{p_{g}}\left(\frac{\sigma}{\mu U_{g}}\right)^{\frac{5}{2}} \ll 1
$$

\section{General Solution}

The approximate solution of equations (54) to (56) given by equations $(74)$ to $(76)$ suggests that if the following nondimensional variables

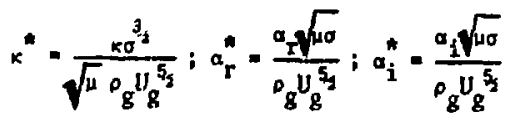

are used in equations (54) to (56), the coefficients of various terms in these equetions become a known function of a viscosity parameter

$$
\left[\left(\rho / \rho_{g}\right)\left(\sigma / \mu U_{g}\right)^{5}\right]
$$

Thus, to obtain a continuous solution of equations (54) to (56) over a wide range of flow parameters, we merely need to vary the viscosity parameter. Such a solution of equations (54) to (56), obtained numerically in terms of above non-dimensional variables, as a function of viscosity parameter is displayed in Fig. 2. Also shown in Fig. 2 are the approximate solutions for very small and very large values of the viscosity parameter. Fig. 2 shows that the high-viscosity liquid solution is valid approximately for

$$
\left(\rho / \rho_{g}\right)\left(0 / \mu U_{g}\right)^{\frac{5}{2}} \leqslant 10^{-2}
$$

and the low viscosity liquid solution is approximately valid for

$$
\left(\rho / \rho_{g}\right)\left(\sigma / \mu U_{g}\right)^{\frac{5}{2}} \geqslant 50
$$

Fig. 2 also shows that the viscosity acts to reduce the amplification as compared to the inviscidIiguid-phase case.

It may be noted that the solutions of equations (54) to (56) presented in the foregoing must satisfy the following inequalities:

$$
\begin{gathered}
\frac{|a|}{k \|_{g}} \ll 1 ; \\
|\ell| a \gg 1 ; \text { ka } \gg 1 ; \text { and }\left|\kappa_{c}\right| a \gg 1 \text {. }
\end{gathered}
$$

In inequality (81), it can easily be shown, by use of equations (25) and (50), that $\left|\kappa_{\mathrm{c}}\right|<\kappa<|\ell|$; therefore, only the third of the foregoing inequalities need to be considered. Expressing the foregoing inequalities in terms of flow parameters for the inviscid-liquid case, with use of equations (64) to (66), inequality (80) becomes

$$
\left(\frac{\rho_{g}}{\rho}\right)^{0.4} \ll 1
$$

and the third of the Inequalities (BI) becomes

$$
a>\frac{\sigma}{F_{g} U^{2}} \text {. }
$$

For a highly viscous 11quid, with the use of equa- 


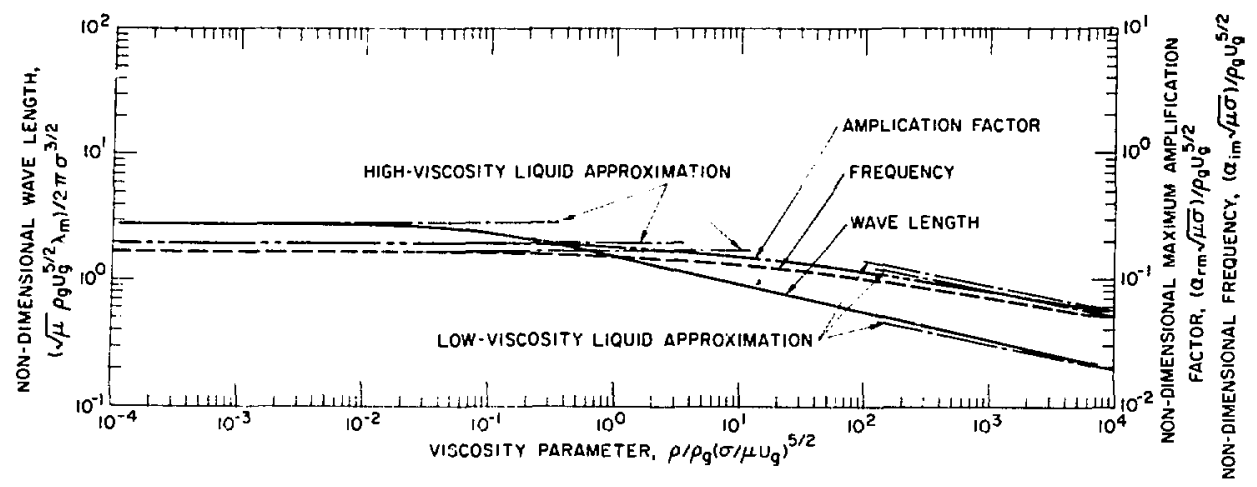

Fig.2 Non-dimensional most probable wave length, frequency, and maximum amplification factor as a function of non-dimensional viscosity parameter

tions (74) to $(76)$, the inequality (80) reduces to

$$
\frac{0}{\mu U_{g}} \ll 1
$$

and the third of the inequalities (81) reduces to

$$
a \gg \frac{d}{\rho \mathrm{g}^{2} \mathrm{~g}} \text {. }
$$

It is clear that a non-dimensional plot of various parameters, such as shown in Fig. 2 , can only be applied if inequalities (80) and (81) are satisfied. It may also be noted that for very heavy gases (e.g., xenon) discharged at very high throat pressures or stagnation pressures into an Inviscid liquid, the inequality (82) is satisfied only very approximately, and the error increases in neglecting terms of order $\left(|\alpha| / \kappa U_{g}\right)$ as the throat pressure goes up.

\section{DROPIET SIZE AND RATE OF IIQUID ENTRAINMENT}

In formulating a model for liquid atomization by a high-velocity gas stream, Mayer (11) postulated that when the amplitude of the windinduced wave on an unstable gas-liquid interface has grown to a dimension comparable with the wave length, $\lambda$, the parts of liquid surface protruding (1.e.. crests of waves) Into gas stream are torm away as "Iigaments" from which droplets of size proportional to wave length are formed. Taylor (12), In the application of the instabllity analysis for the viscous liquid surface under the action of the wind blowing over it to the determination of sizes of drops detached from an unstable fluid surface, postulated that the size of the most frequent arops is proportional to the wave length at the maximum amplification of the wave. Assuming that Mayer's postulated mecisanism for liquid breakup and Taylor's hypothesis for the size of liquid breakup can be extended to Include the process of liquid entrainment at the gasIiquid interface by a high-speed gas jet, then at all locations along the length of the jet where the liquid phase protrudes into the gas jet (i.e., troughs of waves) with an amplitude comparable to most probable wave lergth (i.e., the wave length at maximum amplification of the wave), a ring of liquid of mean radius equal to the radius of the jet itself will be iorn away; the ring thickness is proportional to the most probable wave length. Subsequently, these rings of liquid, being unstable because of a large ratio of surface energy to mass, break up into droplets of size proportional to most probable wave length.

\section{Droplet Size}

If $D_{m}$ is the most probable size (diameter) of the droplets formed, then Taylor's hypothesis implies that

$$
D_{m} \propto \lambda_{m}
$$

On substituting for $\lambda_{m}\left(=2 \pi / k_{m}\right)$ from equation (64) for an inviscld liquid phase, equation (85) becomes

$$
\frac{\rho_{g} U_{g}^{2 D} D_{m}}{\sigma}\left(\frac{\rho}{\rho_{g}}\right)^{0.2}=W\left(\frac{\rho}{P_{g}}\right)^{0.2}=B_{0}
$$

where We is the Weber number and $\beta_{0}$ is a constant. The foregoing result for a sonic gas stream is analogous to that of Hinzes! "critical" Weber number criterion (16,17) for splitting up of the 
drops of inviscid liquids by incompressible air stream.

On substituting for $\lambda_{m}\left(=2 \pi / \kappa_{m}\right)$ from equation (74) for a highly viscous liquid phase, equation (85) takes on the following form:

$$
\frac{\rho_{\mathrm{g}} \mathrm{U}_{\mathrm{g}}^{2} \mathrm{D}}{\sigma}\left(\frac{\mu \mathrm{U}_{\mathrm{g}}}{\sigma}\right)^{\frac{1}{2}}=W_{e}\left(\frac{\mu \mathrm{U}_{\mathrm{g}}}{\sigma}\right)^{\mathrm{L}_{\mathrm{S}}}=\mathrm{B}_{0}^{\circ},
$$

where $B_{0}^{-} \simeq\left(0.803 B_{0} / 0.352\right)$, the constants being obtained from equations (64) and (74).

For liquid of intermediate viscosity [i.e., those liquids which do not satisfy either of inequalities (67) or (77) ], the value of $\lambda_{m}$ in equation (85) should be obtained from the simultaneous solution of equations (54) to (56) as given in Fig. 2 .

Rate of Liguid Entrainment in the Gas Jet

If $I_{0}$ is the initial length of the jet from the orifice in which the disturbance wave with wave length corresponding to maximum amplification on the gas-liquid interface, starting from zero amplitude at $x=0$ (i.e., just at the exit from the orifice where gas jet first comes into contact with the outside liquid), achieves an amplitude $\xi_{m}$ comparable to the wave length, $\lambda_{m i}$, then from equation (6), we obtain

$$
n\left(I_{0}, t\right)=r_{m}\left(t=I_{0} / U_{g}\right)=n_{0} \exp \left(a_{r m} L_{0} / U_{g}\right) .
$$

Expressing amplitudes $\xi_{\mathrm{m}}$ and $\eta_{0}$ in terms of wave length $\lambda_{m}$ by using Taylor's hypothesis, we have

$$
E_{m} \times E \lambda_{m} ; n_{0}=\varepsilon \lambda_{m}
$$

where $E$ and $\varepsilon$ are constants of proportionality, but $E$ is much smaller than $E$, because the initial amplitude, $\eta_{0}$, is very small as compared to the amplitude, $\xi_{m}$, at which the disturbance wave breaks up. The substitution of equation (89) into equation (88) yields

$$
\mathrm{L}_{0}=\ln (E / \varepsilon) \mathrm{U}_{\mathrm{g}} / \alpha_{\mathrm{mm}}
$$

If $L$ is the total length of jet in which entrainment of the liquid is to be considered, then the effective length of jet in which entralnment takes place is ( $\left(\mathrm{I}-\mathrm{I}_{0}\right.$ ). The number of liquid rings (Mayer's hypothesis, as discussed previousIy) torn off from the troughs of waves at maximum amplification by the gas jet is given by $\left(I-I_{0}\right) /$ $\lambda_{m}$, and the amounts of liquid, $M_{l}$, contained in these rings is proportional to

$$
M_{\ell} \propto 2 \pi a \pi\left(\lambda_{m} / 2\right)^{2} \rho\left[\left(L-L_{0}\right) / \lambda_{m}\right]
$$

and the rate of liquid entrainment is proportional to

$$
\dot{H}_{\mathrm{R}} \times 2 \pi a \pi\left(\lambda_{\mathrm{m}} / 2\right)^{2} \rho\left[\left(L-L_{0}\right) / \lambda_{\mathrm{m}}\right] / \tau_{\mathrm{m}},
$$

where $\tau_{m}$ is the time interval in which the wave at maximum amplification grows from initial amplitude $\eta_{0}$ to an amplitude, $\xi_{\mathrm{m}}\left(\tau_{\mathrm{m}}\right)$, at which the wave preaks up. Therefore, this time interval, $\tau_{m}$, between the detachment of successive liquid rings corresponding with the wave length, $\lambda_{m}$, and to an exponential growth of the amplitude, is obtained by use of equation (89) as

$$
\tau_{\mathrm{m}}=\ln (\mathrm{E} / \mathrm{E}) / \mathrm{a}_{\mathrm{m}}
$$

Substituting equation (92) into equation (91), we obtain

$$
M_{\ell} / \rho=Q_{L}=\text { в } 2 \pi a \pi\left(\lambda_{m} / 2\right)^{2}\left[\left(I,-L_{0}\right) / \lambda_{m}\right]^{a} \mathrm{rm} \text {, }
$$

where $\beta$ is a constant of proportionality. Nondimensionalizing the foregoing volumetric rate of liquid entrainment by a standard (i.e., at room temperature and one atmosphere pressure) volumetric rate of gas discharge, $Q_{g a}$, we obtain

$$
\begin{aligned}
& Q_{L} / Q_{g a}=\text { в } 2 \pi a \pi\left(\lambda_{m} / 2\right)^{2}\left[\left(L-L_{0}\right) / \lambda_{m}\right] \times\left[0_{g a} /\left(\pi a^{2} g g^{\prime \prime} g\right)\right] a_{m \pi} \\
& =B \frac{\pi}{2}\left(\frac{L-L_{0}}{a}\right)\left(\frac{\alpha_{m^{\lambda} m}^{\lambda}}{U_{g}}\right)\left(\frac{\rho_{g a}}{\rho_{g}}\right) \text {, }
\end{aligned}
$$

where $\rho_{\text {ga }}$ is the density of gas at standard conditions.

For the inviscid liquid, on substituting from equations (64) and (65) for $\lambda_{\mathrm{m}}$ and $\alpha_{\mathrm{rm}}$, respectively, the foregoing expression becomes

$$
\frac{Q_{L}}{Q_{g a}}=\frac{0.35717694}{0.80327205} \beta \pi^{2}\left(\frac{\rho_{g}}{\rho}\right)^{0.4}\left(\frac{\rho_{g a}}{\rho_{g}}\right)\left(\frac{L-L_{0}}{a}\right),
$$

in which $I_{0}$, giten by equation $(90)$, becomes, on substituting for $\alpha_{\mathrm{rm}}$ from equation (65),

$$
\mathrm{L}_{0}=\frac{\ln (E / \varepsilon)}{0.357176944}\left(\frac{\rho}{\rho_{g}}\right)^{0.2} \frac{\sigma}{\rho_{g} U_{B}^{2}} .
$$

For the highly viscous liquid, on substi- 
Table I Parameters of Gretzinger's Liquid Atomization Experiment Using Sonic Air Jet

\begin{tabular}{|c|c|c|c|c|c|c|c|c|c|c|c|}
\hline $\begin{array}{l}\text { Gretzinger's } \\
\text { test } \\
\text { Identification }\end{array}$ & $\begin{array}{c}\text { Yass flow } \\
\text { rate of } \\
\text { a1r, } \\
\left(1 \mathrm{~b}_{\mathrm{m}} / \mathrm{min}\right)\end{array}$ & $\begin{array}{c}\text { Air nozzle } \\
\text { area, } \\
\left(\text { in. }{ }^{2}\right) \\
\end{array}$ & $\begin{array}{c}\text { Coefficlent } \\
\text { of discharge } \\
\text { for alx } \\
\text { nozzle, } \\
C_{d} \\
\end{array}$ & $\begin{array}{c}{ }^{D} g^{\prime} \\
\left(1 b_{m} / f^{3}\right)\end{array}$ & $\begin{array}{c}\mathrm{U}_{g^{\prime}} \\
(\mathrm{ft} / \mathrm{sec})\end{array}$ & $\begin{array}{c}\text { Mass mean } \\
\text { diameter, } \\
(\mu)\end{array}$ & $\begin{array}{c}\text { ivave } \\
\text { lengtin, } \\
\text { (u) }\end{array}$ & $\frac{\rho}{o_{g}}\left(\frac{g}{\mu U_{g}}\right)^{\prime}$ & $\frac{\mid a_{1}}{k U_{q}}$ & $c^{i a \times 10^{-3}}$ & \\
\hline $20 \mathrm{~B}$ & .3 .874 & 0.3124 & 0.99 & 0.1500 & 1128 & 10.34 & 2.51585 & 2.434 & 0.2361 & 2.2437 & \\
\hline $23 A$ & 1.624 & 0.0124 & 0.99 & 0.2787 & 1128 & 7.3 & 3.31987 & 1.3098 & 0.0432 & 3.957 & \\
\hline $23 \mathrm{~B}$ & 1.173 & 0.0124 & 2.99 & 0.2013 & 1128 & 8.2 & $0 . \$ 1119$ & 1.8134 & 0.0393 & 2.939 & \\
\hline $18 \mathrm{D}$ & 0.437 & 0.0121 & 0.82 & 0.0924 & 934 & 24.6 & 1.07765 & 6.3299 & 0.0325 & 2.358 & 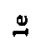 \\
\hline $9 A$ & 1.324 & 0.0121 & 0.82 & 0.2812 & 934 & 9.4 & 0.45699 & 2.0797 & 0.0455 & 6.592 & $\stackrel{N}{E}$ \\
\hline $10 \mathrm{~A}$ & 1.324 & 0.0121 & 0.82 & 0.2812 & 934 & 8.4 & 0.45699 & 2.0797 & $0.345 j$ & 6.592 & \\
\hline $11 \mathrm{~A}$ & 1.324 & .0 .0121 & 3.82 & 0.2812 & 934 & 8.8 & 0.45699 & 2.7797 & 0.0455 & 6.592 & $\stackrel{\vec{z}}{\alpha}$ \\
\hline 98 & 3.931 & 0.3121 & 0.82 & 0.1974 & 934 & 14.4 & 3.59991 & 2.9608 & 0.3411 & 4.755 & \\
\hline 113 & 2.337 & 2.3121 & 0.82 & 0.1974 & 934 & 22.3 & 3.59901 & 2.9608 & $0.04: 1$ & 4.765 & 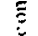 \\
\hline 21.2 & 1.535 & 9.3123 & 2.35 & 0.2837 & 1082 & 8.7 & $1.3 \div 17)$ & 1.4266 & 2.2431 & $: 3.216$ & \\
\hline 218 & 1.372 & 0.0123 & 0.95 & 0.2018 & 1082 & 8.4 & 0.44436 & 2.0054 & 0.0398 & 10.994 & \\
\hline $22 !$ & 1.372 & 0.0120 & 3.95 & 0.2018 & 1082 & 9.3 & 0.44436 & 2.0054 & 0.3398 & 10.994 & \\
\hline $25 \mathrm{~A}$ & 1.793 & 0.0120 & 0.95 & 0.2018 & 1082 & 10.2 & 0.44436 & 2.0054 & 0.0398 & 10.994 & \\
\hline $13 \mathrm{~A}$ & 1.989 & 0.00643 & 0.97 & 0.3676 & 1105 & 6.8 & 0.26861 & 1.0452 & 0.0469 & 10.008 & $\vec{N}$ \\
\hline $14 \mathrm{~A}$ & 1.083 & 0.00643 & 0.97 & 0.3676 & 11.25 & 5.4 & 0.26861 & 1.0452 & 0.3469 & 10.008 & $\cong$ \\
\hline $14 \mathrm{~B}$ & 0.734 & 0.00643 & 2.97 & 0.2376 & 1105 & 7.8 & 0.37636 & 1.6168 & 0.0415 & 6.718 & है \\
\hline $15 \mathrm{C}$ & 0.322 & 0.00043 & 0.97 & 0.1087 & 1105 & 12.8 & 0.68768 & 3.5349 & 3.0329 & 3.273 & 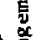 \\
\hline 13D & 0.322 & 0.00643 & 0.97 & 0.1087 & 1105 & 17.5 & 0.68768 & 3.5349 & 0.0329 & 3.273 & 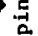 \\
\hline $24 \mathrm{C}$ & 0.476 & 0.00387 & 0.97 & 0.2671 & 1105 & 6.9 & 0.34393 & 1.4387 & 0.0429 & 6.387 & $E$ \\
\hline $25 \mathrm{~A}$ & 1.325 & 0.00920 & 3.97 & 0.3127 & 1105 & 6.3 & 0.30443 & 1.2286 & 0.0448 & 9.812 & 岂 \\
\hline 253 & 1.972 & 0.00920 & 0.37 & 0.2577 & 1105 & 5.4 & 0.35351 & 1.4908 & 0.0425 & 8.223 & $\stackrel{D}{y}$ \\
\hline
\end{tabular}

tuting for $\lambda_{\text {and }} \alpha_{\mathrm{rm}}$ from equations (74) and (75), respectively, equation (94) becomes

$$
\frac{Q_{L}}{Q_{g a}}=\frac{0.19740649}{0.35191129} B \pi^{2}\left(\frac{g}{\mu U_{g}}\right)\left(\frac{\rho_{g a}}{\rho_{g}}\right)\left(\frac{L-L_{0}}{a}\right),
$$

in which $L_{0}$, given by equation (83), now becomes

$$
L_{0}=\frac{\ln (E / \varepsilon)}{0.19740649}\left(\frac{\mu U_{g}}{\sigma}\right)^{\frac{1}{2}} \frac{\sigma}{\rho_{g}^{U_{g}^{2}}} .
$$

VERTFICATION OF CORRELATIONS FOR DROPLET SIZE AND RATE OF LIQUDD ENTRAINMENT BY COMPARISON WIT'भ EXPERIMENTAI. DATA

The approach to the problem of deternining droplet size formed and the rate of liquid entrainment at the unstable gas-liquid interface through the use of stability theory can, at best, yield results to an order of magnitude by providing proportionality relationships (85) and (94), respectively. The constants of proportionality must ultimately be determined by correlating the experimental data on the basis of these proportionality relationships.

\section{Correlation for Droplet Size}

The experimental data utilized for the purpose of obtaining the constant of proportionality between droplet size and wave length [see equation $(85)]$ are those of Gretzinger (18). He studied pneumatic atomizers in which a liquid is disintegrated into droplets by action of high-velocity air. The atomizers usec for obtaining data of present interest were of two types. The first one, called convenging type, consisted of two concentric nozzles, in which the inner liquid nozzle was surrounded by a concentric air nozzle. The second, called impingement type, was also made up of two concentric nozzles; the inner nozzle carries the air; the liquid to be atomized is carried in the annular space between the two nozzles. In addition, an impinger, mounted on a 1/16-in.-dia rod was suspended from a support mounted inside the alr nozzle. A distance of $1 / 4 \mathrm{in}$. was maintained between the outlet of the air nozzle and the impinger. The air velocity was varied from 


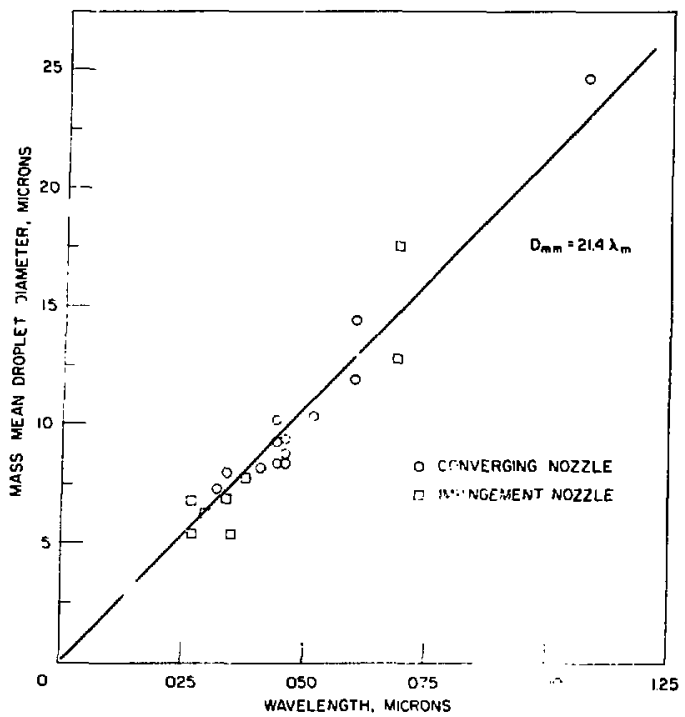

Fig. 3 Mean proportionality relationship between mass mean aroplet diameter and most probable wave length. The points denote the measured values of mass mean droplet diameters by Gretzinger

near sonic to sonic velocity. The data utilized for the present analysis, are for the sonic velocicy of ail and very low liquid-jet velocities. The relevant parameters are given in Tabie 1 . The values for the viscosity parameter given in the table indicate that the viscosity terms in the solution of equations (54) to (56) must be retained. It must be noted that the geometrical arrangement for the gas-liquid system used in Gretzinger's impingement-nozzle experiment is approximately similar to the conriguration of a fission-gas jet impinging on the opposite pin and the liquid coolant surrounding the jet. Fig. 3, which shows the plot of the aroplet size (represented by the mass mean diameter) against the most probable wave length, indicates that there exists very satisfactory linear proportionality between measured droplet size and the most probable wave length, thus verifying Taylor's postulate concerning droplet size formed. It also shows that in the short-wave approximation, the gas-liquid arrangements in the two types of atomizers are approximately equivalent; furthermore, the presence of impinger has negligible effect on the mass mean droplet size. The criterion for the applicablilty of short-wave approximations, $\left|\kappa_{c}\right| a \gg 1$, to Gretzinger's data is based on the radius of liquid jet in the converging nozzle and the radius of a ix jet in the impingement nozzle. Table 1 shows that this shortwave approximation criterion is satisfied. In ad-

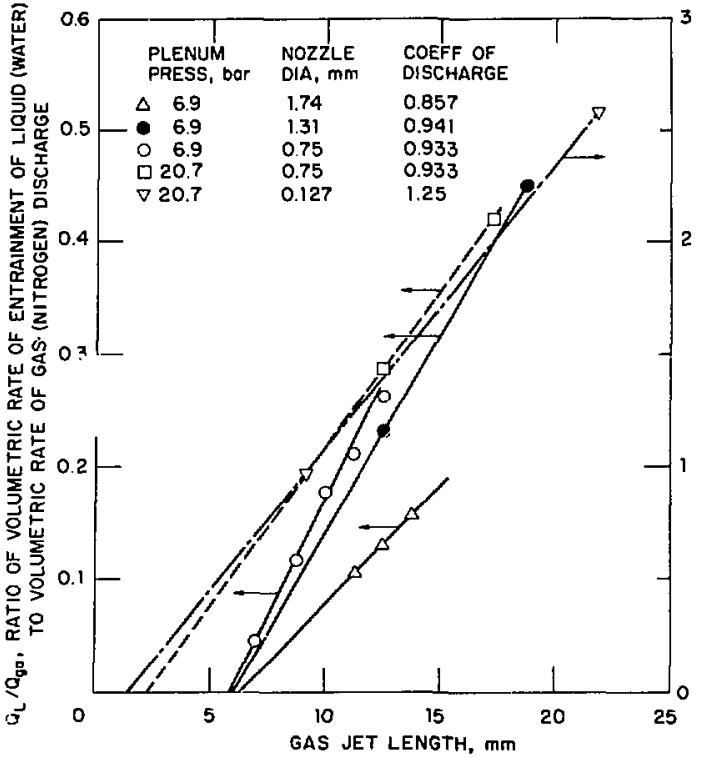

Fig. 4 Measured ratio of volumetric rate of liquid (water) entrainment to volumetric rate (reduced to room temperature and atmospheric pressure) of gas (nitrogen) flow in a jet as a function of jet length by Bell et al.

dition, Table 1 shows that inequality (80) is satisfied.

\section{Correlations for Rate of Iiquid Entrainment} and Initial Non-entrainment Length

The only experimental data available on the rates of liquid entrainment and initial nonentrainment lengths are those of Bell et al. (19). The rates of liquid entrainment as a function of the jet length with stagnation pressure and nozzle diameters, together with respective coefficients of discharge for the nozzles, are shown in Fig. 4. The experiment was performed at room temperature. The initial non-entrainment lengths were obtained by the linear extrapolation of the data shown in F॰5. 4. In Fig. 5 is shown the non-dimensional plots of rates of liquid entrairment obtained from Fig. 4 against the non-dimensionai parameter according to equation (94); the amplification factors, $\alpha_{\mathrm{rm}}$, and wavelength $\lambda_{\mathrm{m}}$ that occur in the "independent" non-dimensional parameter of Fig. 5 were obtained for the flow parameters given in Fig. 4 from the solution of equations (54) to (56). For the range of flow parameters of the experiment, the range of values for $\left|\kappa_{c}\right| a$ was found to be 1150 to 4000 , and for $|\alpha| / k U_{\text {, }}$ to be 0.054 to 0.075 , thus implyins that inequalities (80) and (81) are satisfled and the approximations implied in the deri- 


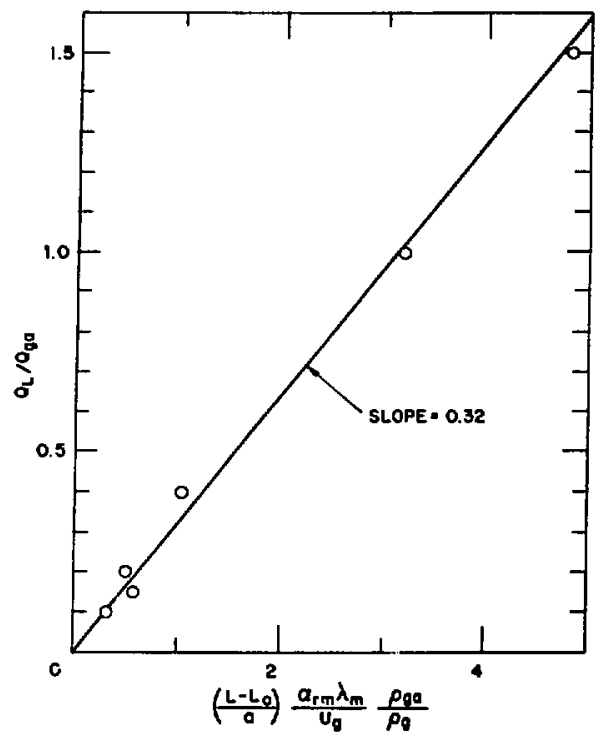

Fig.5 Mean proportionality relationship between non-dimensional volumetric rate of liquid entrainment and non-dimensional parameters defined by equation (94). The points denote measured values of ratios of volumetric rate of liquid (water) entrainment to volumetric rate (at atmospheric pressure and room temperature) gas flow in the jet, by Bell, et al.

vation of equations (54) to (56) are valid. In Fig. 5, it can be seen that a linear least-squares fit through the marked points and containing the origin can be made, thus verifying the proportionality relationship between the rates of liquid entrainment and the non-dinensional parameter of equation (94).

In Fig. 6 is shown the plot of initial nonentrainment length obtained from Fig. 4 by linear extrapolation of the data against the ratio, $\mathrm{U}_{\mathrm{g}} / \alpha_{\mathrm{rm}}$. Once again, we can approximately fit a least-squares straight line containing the origin, thus verifying the proportionality relationship (90).

\section{APPLICATION TO LMFBR REACTOR}

For application to an LMFBR reactor, we consider the applicability of the low-viscosity liquid approximations for which the inequality (79) must be satisfled for the range of flow parameters of interest. The following representative values for the parameters occurring in this inequality are chosen:

1 Gas-Plenum temperature $=1100 \mathrm{~F}$

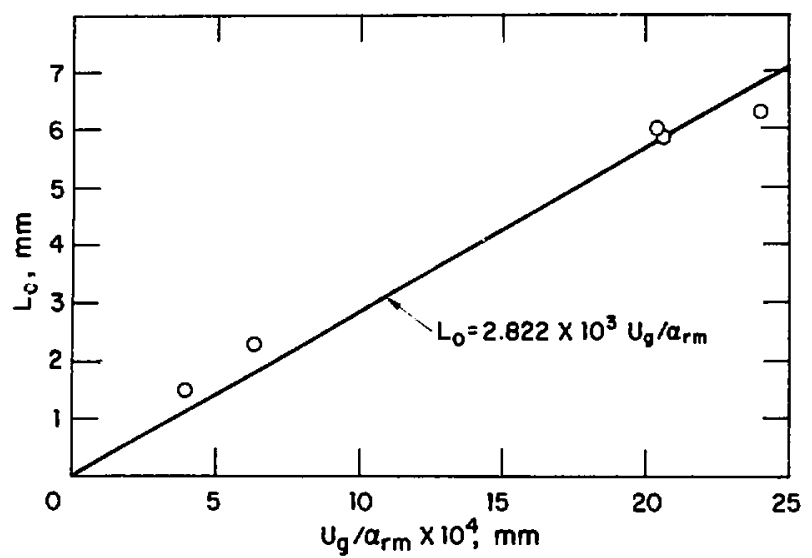

Fig.6 Mean proportional1ty relationship between non-entrainment length of a gas jet and parameter defined by equation ( 90$)$. The points denote values of initial non-entrainment lengths obtained by extrapolating Iinearly the data by Bell, et al. presented in Fig. 4

2 Initial gas-plenum pressure $=800 \mathrm{psia}$

3 Density of Ilquid-metal coolant at core exit temperature of $1100 \mathrm{~F}, \rho=50.553$ $1 b_{m} / c u$ ft

4 Surface tension at $1100 \mathrm{~F}, \sigma=0.01 \mathrm{lb} / \mathrm{ft}$

5 Viscosity at $1100 \mathrm{~F}, \mu=1.39972 \times 10^{-4^{4}}$ $1 b_{m} / f t-s e c$

6 Fission-gas density at the throat conditions ccrresponding to gas plenum pressure of $\sim 800$ psia and temperature of $1100 \mathrm{~F}, \rho_{g}=4.07$ $1 b_{m} / c u$ ft

7 Gas velocity at the throat condition: corresponding to gas plenum temperature of $1100 \mathrm{~F}, \mathrm{U}_{\mathrm{g}}=860 \mathrm{fps}$.

For the values of the parameters given in the foregoing, the viscosity parameter in inequality (79) is approximately equal to 55.0. From inequality (79), It is clear that the value of the viscosity parameter increases as pressure in the plenum decreases, and thus the applicability of the low-viscosity liquid approximation becomes more valid for low pressures in the plenum.

\section{CONCLUSIONS}

The present investigation analyzes the Kelvin-Helmholtz instability of the gas-liquid interface of a cylindrical sonic gas jet, emerging from a vessel with plane walls and submerged in an infinite mass of a viscous liquid, under the accion of the pressure perturbations, interfacial 
surface tension, and finite liquid viscosity by the method of small perturbations.

The use of short-wave approximations (relevant to problems of atcmization and droplet entrainment by high-speed gas stream), together with low-viscosity-liquid approximations, yielded expressions (64) to (66) for the growth of the instability at maximum rate. These expressions are valid approximately for a viscosity parameter $\left(\rho / \rho_{g}\right)\left(\sigma / \mu U_{g}\right)^{5 / 2} \geqslant 50$. For highly viscous liquids, the growth of instability at maimum rate is governed by expressions (74) to (76), which are valid for values of the viscosity parameter $\left(\rho / \rho_{g}\right)(\sigma /$ $\left.\mu \mathrm{U}_{g}\right)^{5 / 2} \leqslant 10^{-2}$. For the viscosity parameter $10^{-2}<\left(\rho / \rho_{g}\right)\left(0 / \mu U_{g}\right)^{5 / 2}<50$, equations (54) to (56) should be solved for a given value of viscosity parameter or Fig. 2 should be used, using viscosity parameier as an independent variable.

For waves having most rapid rate of growth, Taylor's and Mayer's hypotheses, together with data of Bell et al., provided the following relationships for the rate of liquid entrainment and the initial non-entrainment length: For rate of liquid entrainment,

$$
\frac{Q_{f}}{Q_{g a}}=0.313\left(\frac{L-L_{0}}{a}\right) \frac{{ }^{a} m^{2} m}{U_{g}}\left(\frac{\rho_{g a}}{\rho_{g}}\right) ;
$$

for non-entraiment length,

$$
L_{0}=2.822 \times 10^{3} U_{g} / \alpha \mathrm{sm}
$$

The use of Taylor's hypothesis and Gretzinger's data provided the relationship ror the droplet size (represented by mass mean diameter, $\mathrm{D}_{\mathrm{mm}}$ ) as

$$
l_{\mathrm{nm}}=21.4 \lambda_{\mathrm{m}} .
$$

It is demonstrated that for the range of parameters pertining to an LMFBR reactor, the low-viscosity liquid approximations are valid. The application of the present resuits to the determination of heat-transfer coefficient for the spray-cooled region of the gas jet-impingement area of the fuel pin in an LMFBR reactor using non-vented fuel pins is given in reference (20).

\section{ACKNOWLEDGMENTS}

The discussions with $J$. B. van Erp on various aspects of the analysis are deeply appreciated. The author would like to thank H. F. Fauske for the very helpful remarks that he has made on the subject matter of this paper. The assistance af- forded by $\mathrm{C}$. Fiala in carrying out the romputations is deeply appreciated.

The work was performed under the auspices of the U. S. Atomic Energy Commission.

\section{REFERENCES}

1 Chawla, T. C., and Hogluni, B. M., "A Study of Coolant Transients During a Rapid Fission Gas Release in a Fast Reactor Subassembly, "Nuclear Science and Engineering, Vol. 44, 1971, p. 320 .

2 Chang, I. D., and Russell, P. E., "Stability of a Liçuid Layer Adjacent to a High-Speed Gas Stream," The Physics of Fluids. Vol. 8, 1964 , p. 1018 .

3 Nachti heim, P. R., "Stability of Crosshatched Wave Patterns in Thin Liquid Films Adjacent to Supersonic Streams," The Ploysics of Fluids, Vol. 13, 1970, p. 2432.

4 Nayfel, A. H., and Saric, W. S., "Nonlinear Kelvin-Helmholtz Instability," Journal of Fluid Mechanics, Vol. 46, 1971, p. 209.

5 Draxin, P. G., "Kelvin-Helmholtz Instability of Finite Amplitude," Journal of pluid Mechanics, Vol. 42, 1970, p. 321.

6 Hewitt, G. F., and Hall-Taylor, N. S., Annular Two-Phase Flow, Pergamon Press, Oxfcrd, 1970.

7 Powell, A., "On the Mechanism of Choked Jet Noise," Proceedings of Physical Society of Iondon, Vol. B66, $1953, \mathrm{p} .1039$.

8 ovsiannikov, L. V., "Gas Flow With Straight Transition Iine," NACA TM-1295, 1951.

9 Sedov, I. I., Two-Dimensional Problems in Hydrodynamics and Aerodymamics, Wiley, New York, 1951.

10 Tam, C. K. W., "Directional Acoustic Radiation From a Supersonic Jet Generated by Shear Layer Instability," Journal of Fluid Mechanics, Vol. 46, 1971, p. 757 .

11 Mayer, E., "Theory of Liquid Atomization in High Velocity Gas Streams," Journal American Rocket Society, Vol. 31, 1961, p. 1783.

12 Taylor, G. I., "Generation of Ripples by Wind Blowing Over a Viscous Fluid," The Scientific Papers by G. I. Taylor, G. K. Batchesor, ed., Vol. 3. 1940.

13 Wilson, R. E. et al.. "Experimental

Evaluation of Fission-Gas Jet-Impingement in LMFBR Subassemblies," Paper to be presented at ANS Meeting, Las Vegas, Nev., June 1972.

14 Ievich, G. , Phys Icochemical Hydrodynamics, Prentice-HalI, Iondon, 1962.

15 Abramowitz, M., and Stegun, I. A., llandbook of Mathematical Functions, Dover Publications, New York, 1965. 